\title{
Sawflies from Külsö-Somogy, South-West Hungary (Hymenoptera: Symphyta)
}

\author{
ATtILA HaRIS \\ H-1076 Budapest, Garay street 19 2/20., Hungary \\ e-mail: attilaharis@yahoo.com
}

Haris, A.: Sawflies from Külsö-Somogy, South-West Hungary (Hymenoptera: Symphyta).

Abstract: 119 sawfly species were reported from Külsö-Somogy. Seven rare species were captured: Aprosthema austriacum (Konow, 1892), Dolerus (Poodolerus) quadrinotatus (Bíró, 1884), Birka (Birka) annulitarsis (Thomson, 1870), Empria hungarica (Konow, 1895), Harpiphorus lepidus (Klug, 1818), Hinatara nigripes (Konow, 1907) and Pachynematus (Larinematus) imperfectus (Zaddach, 1876). New color variation of Dolerus (Poodolerus) quadrinotatus (Bíró, 1884) is described.

Keywords: Hymenoptera, Symphyta, fauna, Külső-Somogy, Hungary, Dolerus quadrinotatus (Bíró, 1884), new color variation

\section{Introduction}

The first species was reported from this region as early as 1900 (MocsáRY, 1900). Látrány Puszta Nature Reserve Area, investigated in 2003, is also part of this region (HARIs, 2003). 87 Symphyta species were reported from here. Haris, 1998, 2001 and Roller and Haris, 2008 also contain high number of sawfly species from Külsö-Somogy (Fig. 1); finally, sporadic data we may find in MóczÁr and ZoMBori 1973; ZomborI 1982 and 1990.

\section{Methods and material}

We spent 15 days in April and 5 days in May and 1-1 days in June and August, with collecting on 41 sampling sites.

The early collecting season started very adversely reasoned by the extreme weather conditions. There was a snowfall at the end of March (although in the mid of January, the temperature reached even the plus $11^{\circ} \mathrm{C}$ ), furthermore early April was rainy. Therefore the species richness was low. We collected 496 specimens of 95 species. Our collection was augmented by earlier captured and still unpublished specimens collected by Zsolt Józan and György Rozner, few specimens by Gyula Sáringer and Ottó Merkl. This additional material consists of 115 specimens of 62 species.

For identification, Zhelochovtsev's work on the sawflies of the European part of the former USSR (ZHELOCHOVTSEV 1988) was consulted. We also used some recent revi- 
sions and works to make the identifications even more precise (BLANK \& RITZAU 1998, HARIS 2006, Koch 1988, ZOMBORI 2016).

For the discussion of the distribution of sawflies, we consulted the book of Roller and Haris titled Sawflies of the Carpathian Basin, History and Current Research (RoLleR \& HARIS 2008), the most recent European checklist of species (TAEGER et al. 2006) augmented by other faunistic records from the Carpathian Basin (GYURKOVICS and HARIS 2012, Haris 2009, 2011, Roller 1993, 1994, 1996, 1998, 1999a, b, c, d, e, 2000, b, c, 2001, 2004,2005, 2006a, b, RoLLER and LuKÁš 1999, RolLEr et al. 2006).

The higher classification of sawflies applied in this work follows the Hymenoptera part of Fauna Europaea (ACHTERBERG 2013).

The dominant collection method was the sweep netting. It was completed in May with individual collection of sawflies.

\section{List of sampling sites in 2018}

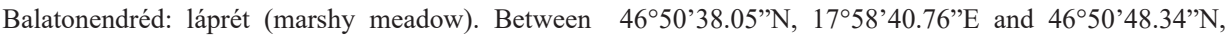
1758'38.86’'E. Altitude: 119-125 m.

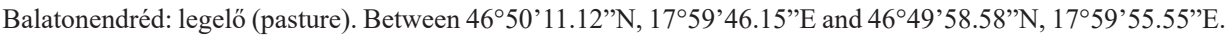
Altitude: 143-184 m.

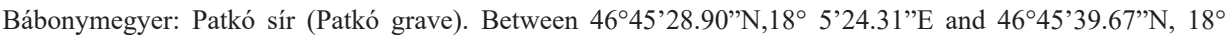
5'23.40"E. Altitude: 165-172 m.

Bábonymegyer: pasture with willows. Between $46^{\circ} 45^{\prime} 3.47^{\prime} \mathrm{N}, 18^{\circ} 77^{\prime} 28.62^{\prime \prime} \mathrm{E}$ and $46^{\circ} 44^{\prime} 54.67^{\prime \prime} \mathrm{N}, 18^{\circ}$ 7'50.38'E. Altitude: 127-153 m.

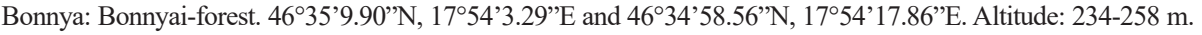

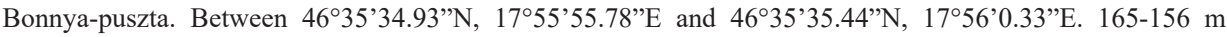
Altitude.

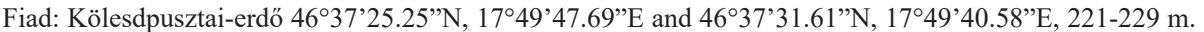

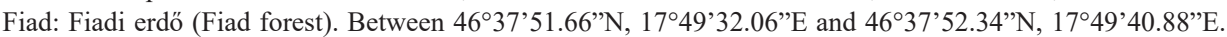
Altitude: $186-189 \mathrm{~m}$.

Hetes: meadow next to the cementery. $46^{\circ} 24^{\prime} 50.73^{\prime \prime} \mathrm{N}, 17^{\circ} 42^{\prime} 21.91^{\prime \prime} \mathrm{E}$ and $46^{\circ} 24^{\prime} 42.77^{\prime \prime} \mathrm{N}, 17^{\circ} 42^{\prime} 13.03^{\prime \prime} \mathrm{E}$ Altitude: $146-148 \mathrm{~m}$.

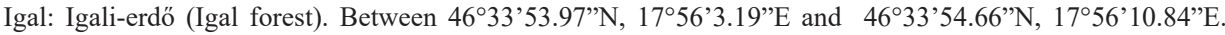
Altitude: $272 \mathrm{~m}$.

Kapoly: Kis-Koppány valley, marshi meadow, alongside the railway between $46^{\circ} 43^{\prime} 3.09^{\prime \prime} \mathrm{N}, 17^{\circ} 58^{\prime} 8.51^{\prime \prime} \mathrm{E}$ and $46^{\circ} 42^{\prime} 53.43^{\prime \prime} \mathrm{N}, 17^{\circ} 58^{\prime} 24.14$ 'E, $167-170 \mathrm{~m}$ altitude.

Kaposfüred: fenyő plantation. Between $46^{\circ} 24^{\prime} 10.09^{\prime \prime} \mathrm{N}, 1^{\circ} 46^{\prime} 32.06^{\prime \prime E}$ and $46^{\circ} 24^{\prime} 11.43^{\prime \prime} \mathrm{N}, 17^{\circ} 46^{\prime} 39.83^{\prime \prime} \mathrm{E}$. Altitude: 161-162 m.

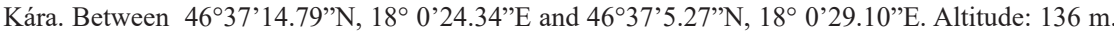

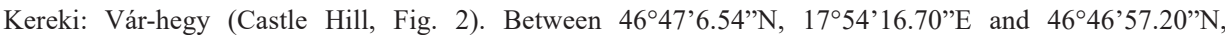
1754'10.78’'E. Altitude: 171-253 m.

Kisbárapáti: láprét (marshy meadow). Between 46³6'55.44”N, 1751'25.38'E and 46³6'53.49”N, 1751'17.20''E. Altitude: 151-153 m.

Magyaregres: Varga-bonyi árok. 46 $27^{\prime} 18.97^{\prime \prime} \mathrm{N}, 1^{\circ} 46^{\prime} 9.16^{\prime \prime} \mathrm{E}$ and $46^{\circ} 27^{\prime} 45.14^{\prime \prime} \mathrm{N}, 17^{\circ} 45^{\prime} 53.53^{\prime \prime} \mathrm{E}$. Altitude: 133-136 m.

Mernyeszentmiklós: forest. $46^{\circ} 33^{\prime} 16.99^{\prime \prime} \mathrm{N}, 1^{\circ} 47^{\prime} 35.58^{\prime \prime} \mathrm{E}$ and $46^{\circ} 33^{\prime} 22.94^{\prime \prime} \mathrm{N}, 1^{\circ} 47^{\prime} 30.88^{\prime \prime} \mathrm{E}$. Altitude: 213-218 m.

Mezőcsokonya: Csokonyai forest $1 \mathrm{~km}$ from the end of Táncsics street. Between $46^{\circ} 24^{\prime} 32.60^{\prime \prime} \mathrm{N}, 1^{\circ} 38^{\prime} 33.27^{\prime} \mathrm{E}$ and 46 $24^{\prime} 20.38^{\prime}$ N, $17^{\circ} 38^{\prime} 28.00^{\prime}$ 'E. Altitude: 174-185 m.

Mezőcsokonya: Alsónyíres: Csárdahely. Between 46²4’35.44’'N, 17³8'55.17'E and 46²4’22.13”N, 17³9’6.47’'E. Altitude: 173-174 m. 


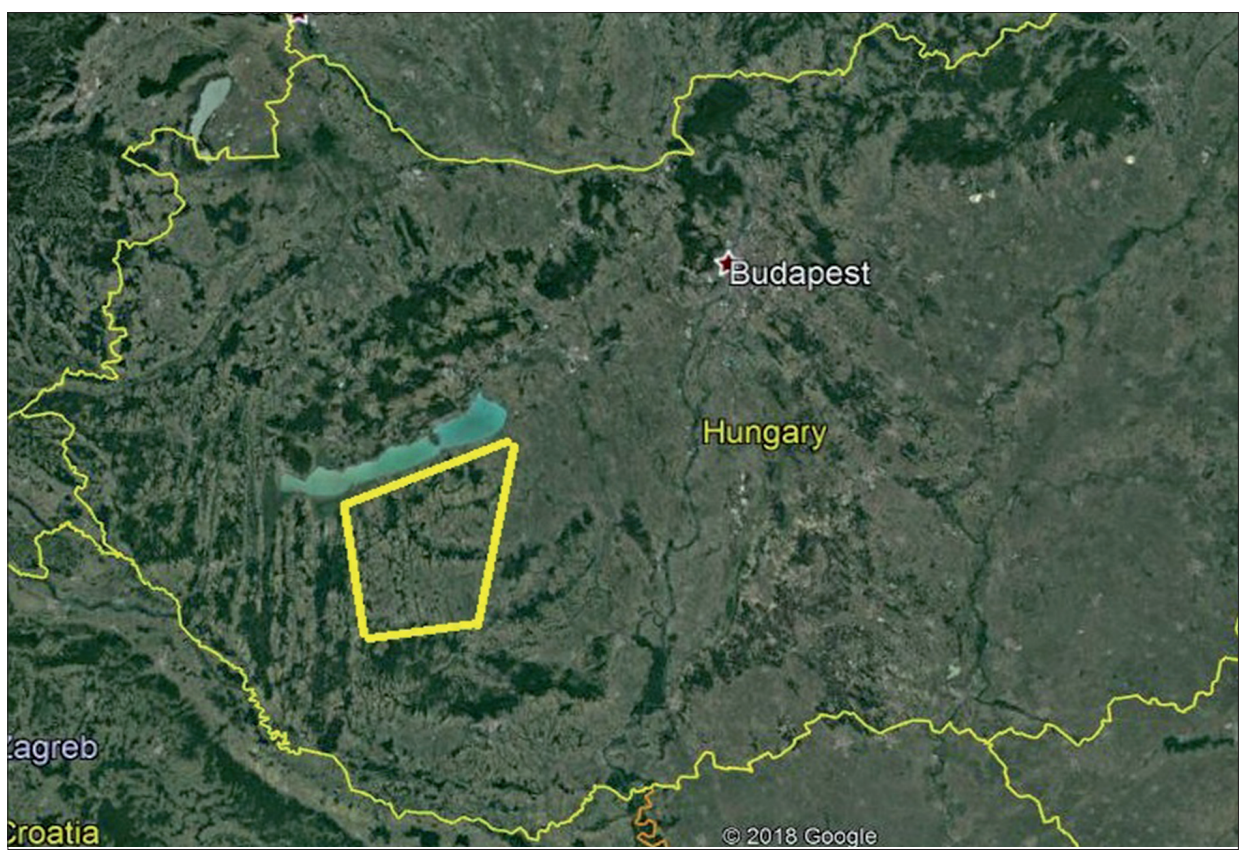

Fig. 1.: The investigated area in Hungary

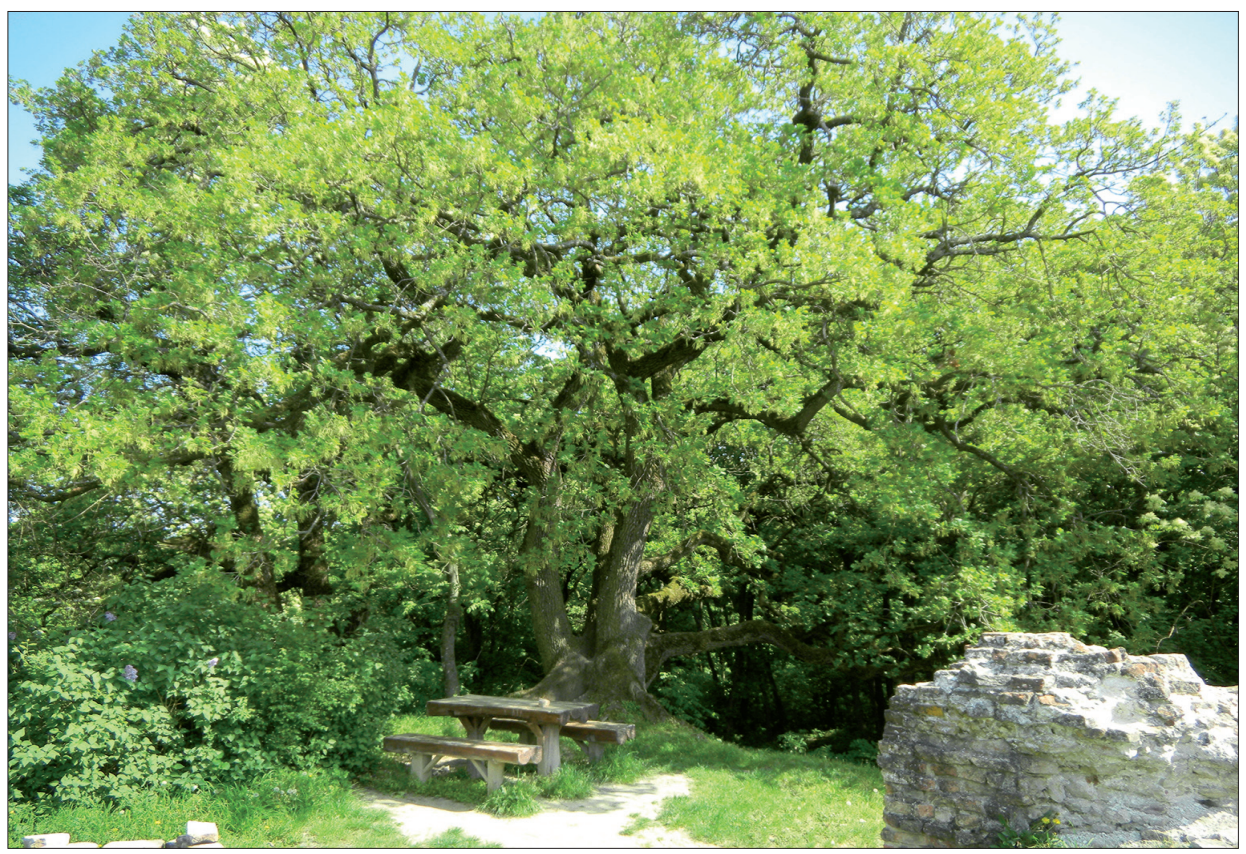

Fig. 2: Kereki: Várhegy (Castle Hill) 


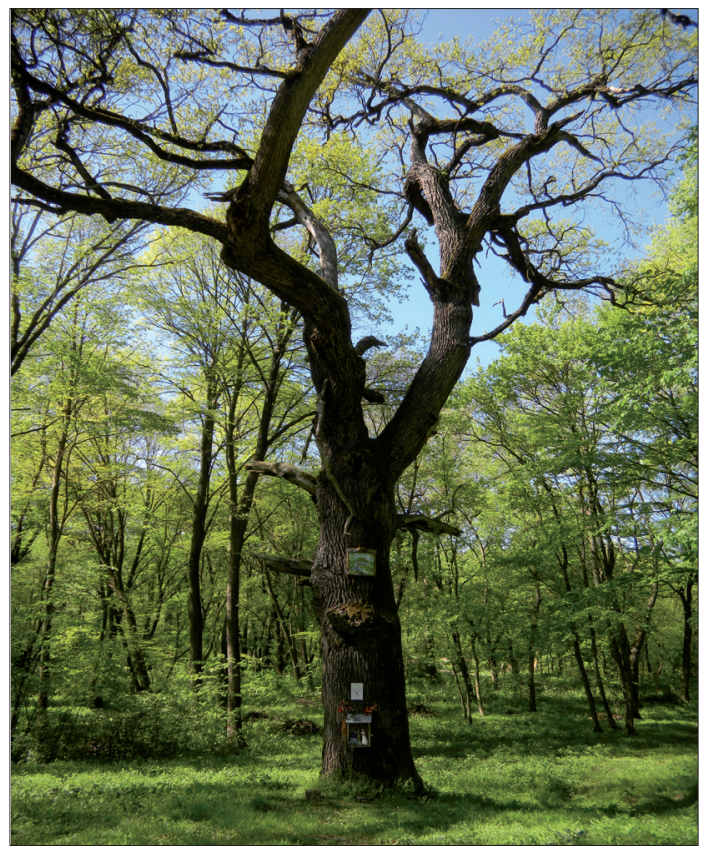

Fig. 3: Ságvár: Képes fa

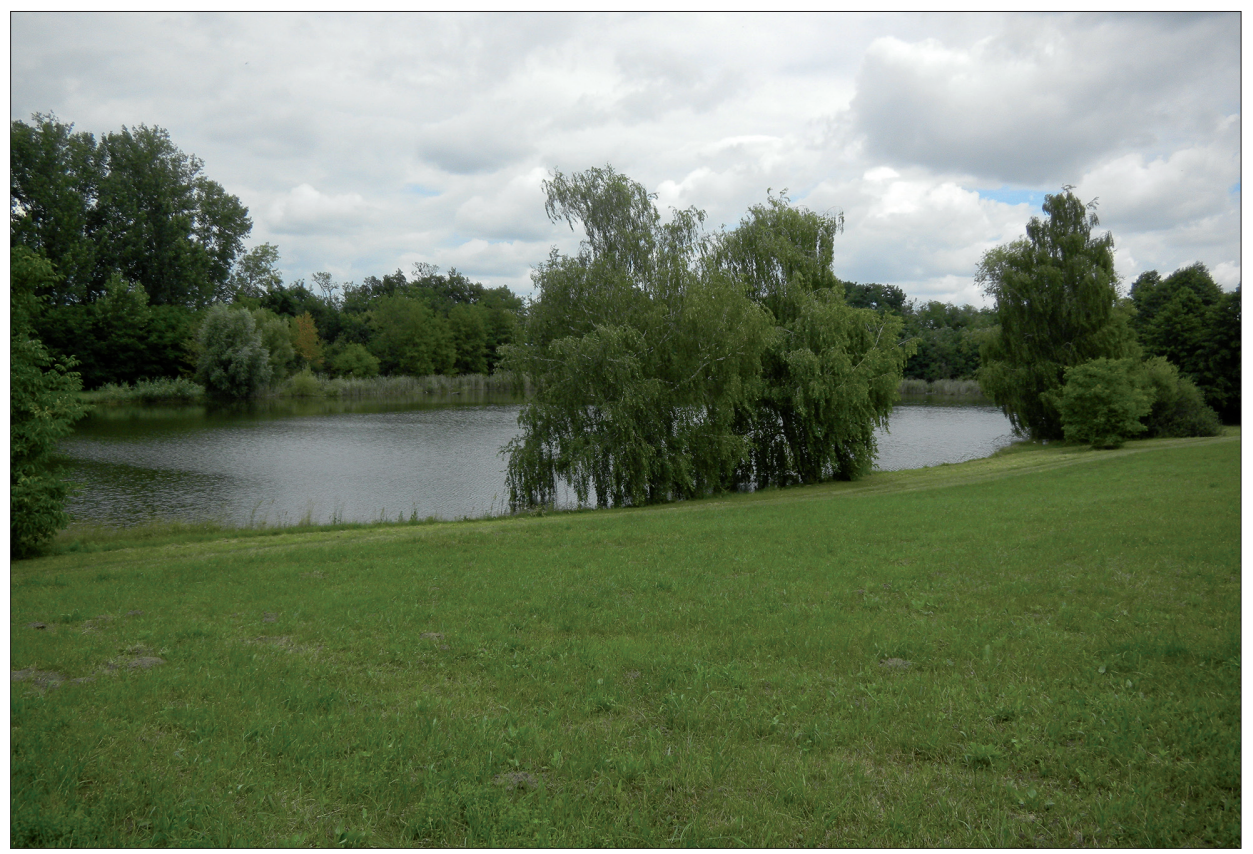

Fig. 4: Somogysárd: Fishing pond 
Miklósi. Dirt road behind the Church of Saint John the Baptist. Between 46³8'45.31' N, 1759'16.17'E and 46³8'43.40”N, 1759'6.02”'E. 191-205 m altitude.

Patalom: Patalomi forest behind elderly home. Between $46^{\circ} 26^{\prime} 47.95^{\prime} \mathrm{N}, 17^{\circ} 55^{\prime} 52.98^{\prime \prime} \mathrm{E}$ and $46^{\circ} 26^{\prime} 35.31^{\prime \prime} \mathrm{N}$, 1756'4.83’E. Altitude: 162-167 m.

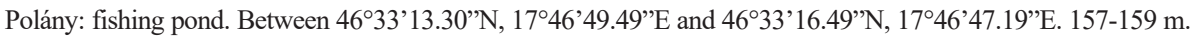
Pusztaszemes: forest opposite of Kossuth street 4. Between $46^{\circ} 46^{\prime} 42.19^{\prime \prime} \mathrm{N}, 17^{\circ} 55^{\prime} 9.14^{\prime \prime} \mathrm{E}$ and $46^{\circ} 46^{\prime} 32.27^{\prime \prime} \mathrm{N}$, 1755'5.47’'E. Altitude: $181-210 \mathrm{~m}$.

Pusztaszemes: Szemesi erdő, behind Petőfi street. Between $46^{\circ} 45^{\prime} 47.07^{\prime \prime} \mathrm{N}, 17^{\circ} 55^{\prime} 30.81^{\prime \prime} \mathrm{E}$ and $46^{\circ} 45^{\prime} 51.07^{\prime \prime} \mathrm{N}$, 1755'21.33'"E. Altitude: 197-207 m.

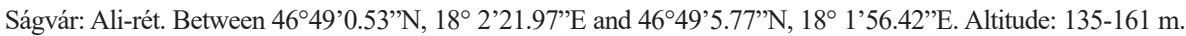

Ságvár: Képes fa (Sacred tree, Fig. 3) and Bögöcsei-legelö (Bögöcse pasture). 46²49'15.84'N, 18 3'12.21'”E and 4649'37.67'N, 18 3'11.91''E. Altitude: 137-165 m.

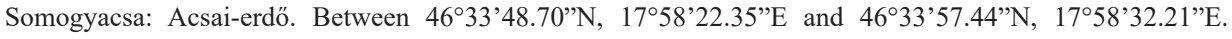
Altitude: $288-300 \mathrm{~m}$.

Somogyaszaló: Deseda. Between $46^{\circ} 26^{\prime} 28.55^{\prime \prime} \mathrm{N}, 17^{\circ} 47^{\prime} 50.76^{\prime \prime}$ E and 46²6’27.25”N, $17^{\circ} 48^{\prime} 9.25^{\prime}$ 'E. Altitude: 142-163. m.

Somogybabod: Somogybabodi-erdő. Between $46^{\circ} 40^{\prime} 46.38^{\prime \prime} \mathrm{N}, 17^{\circ} 45^{\prime} 42.50^{\prime \prime} \mathrm{E}$ and $46^{\circ} 40^{\prime} 43.68^{\prime} \mathrm{N}$, 1745’33.24’'E. Altitude: 175-194 m.

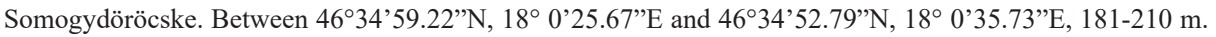

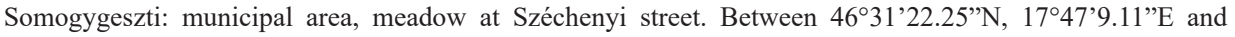
$46^{\circ} 31$ '21.05’'N, 1747'16.79’'E. 146-159 m.

Somogymeggyes: meadow, 46 42 ' 57.74 'N, $17^{\circ} 56^{\prime} 9.34^{\prime \prime}$ E and $46^{\circ} 42^{\prime} 41.38^{\prime \prime} \mathrm{N}, 1^{\circ} 56^{\prime} 25.60$ 'E. Altitude: 184$192 \mathrm{~m}$.

Somogysárd: Fishing ponds (Fig. 4). Between $46^{\circ} 25^{\prime} 14.85^{\prime} \mathrm{N}, 17^{\circ} 36^{\prime} 21.58^{\prime} \mathrm{E}$ and $46^{\circ} 25^{\prime} 7.15^{\prime \prime} \mathrm{N}$, 17³6'27.94'"E. Altitude: 165-172 m.

Somogytúr: Túri-erdő Between 46³9'59.49’N, 1743'49.73’E and 46³9'50.00”N, 1743'53.49”E. 262-264 $\mathrm{m}$ altitude.

Somogytúr: Hárshegyi vadászház. Between 4642’5.62”N, 1743’17.71'E and 4642’7.20”N, 1743'14.96”'E. Altitude: $218-220 \mathrm{~m}$.

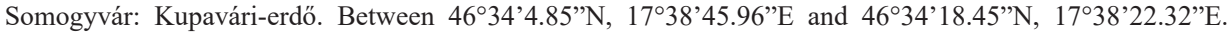
Altitude: $137-180 \mathrm{~m}$.

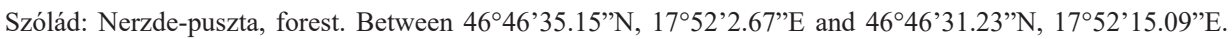
Altitude: 160-201 m.

Szorosad: Koppány brook: at the bridge. Between $46^{\circ} 35^{\prime} 58.07^{\prime} \mathrm{N}, 18^{\circ} 11^{\prime} 31.30^{\prime \prime} \mathrm{E}$ and $46^{\circ} 35^{\prime} 55.14^{\prime \prime} \mathrm{N}, 18^{\circ}$ 1'24.17'E. Altitude $126 \mathrm{~m}$.

Tab: meadow at Koppány brook. Between $46^{\circ} 44^{\prime} 18.08^{\prime \prime N}, 18^{\circ} 4$ '13.36”E and $46^{\circ} 44^{\prime} 14.19^{\prime \prime} \mathrm{N}, 18^{\circ} 4^{\prime} 0.48^{\prime \prime} \mathrm{E}$, 135-137 $\mathrm{m}$ altitude.

Tengöd: Tengödi-forest. Between 4643'29.69"N, $18^{\circ}$ 5'33.15"E and 4643'20.92”N, $18^{\circ}$ 5'58.29"'E. Altitude: 236-263 m.

Törökkoppány: Koppányi forest: Foresters monument: 46³4'19.90”N, 18²'59.19'"E and 46³4'19.59”N, $18^{\circ}$ 3'2.63'"E 179-180 m altitude.

\section{List of species}

\section{Xyelidae}

Xyela graeca Stein, 1876: Látrány: Birkás-legelő, 04. 05. 2002, 1 female. Sporadic. Larva on Pinus nigra.

\section{Pamphiliidae}

Neurotoma nemoralis (Linné, 1758): Igal: Andocsi úti erdő, 14. 04. 2018, 1 female, 1 male. Known hostplants: Prunus mahaleb, P. armeniaca, P. spinosa and P. cerasus. Locally frequent pest. 
Pamphilius sylvaticus (Linné, 1758): Kapoly: Kis-Koppány valley, 26. 04. 2018, 1 female. One of the commonest pamphilid sawfly species. Hostplants: Sorbus aucupariae, Malus spp., Prunus spp. and Crataegus spp.

Pamphilius vafer (Linné, 1767): Somogysárd: Fishing ponds, 23. 04. 2018, 1 male, 25. 04. 2018, 1 male. Sporadic. Known hostplants: Alnus glutinosa, Alnus incana, Alnus fruticosa, Betula pendula and Betula pubescens.

\section{Megalodontesidae}

Megalodontes plagiocephalus (Fabricius, 1804): Balatonendréd: Endrédi-dombok, 06. 05. 2018, 2 females, 1 male. One of the most frequent Megalodontesidae species. Known hostplant: Peucedanum alsaticum.

Megalodontes fabricii (Leach, 1817): Balatonszemes: Bagó-domb, 10. 06. 2008, 1 male. Sporadic. Hostplant unknown.

\section{Argidae}

Aprosthema austriacum (Konow, 1892): Hetes: meadow at the cementery, 27. 04. 2018, 1 male. Rare. Hostplant unknown.

Arge cyanocrocea (Forster, 1771): Bonnya: Bonnyai-forest, 24. 04. 2018, 1 male, 12. 05. 2018, 1 male; Igal: Andocsi úti forest, 12. 05. 2018, 1 female; Kereki: É. Belterület, 29. 06. 2012, 1 female. Common species. Known hostplants: Rubus idaeus and Sanguisorba officinalis.

Arge enodis (Linné, 1767): Somogygeszti: belter., 21. 05. 2007, 1 male; Ecseny: belter, 28. 06. 2006, 1 female; Kánya: belter., 20. 07. 2009, 1 female. One of the commonest Argid sawfly in Hungary, recently sporadically collected. Hostplants: Salix spp.

Arge melanochra (Gmelin, 1790): Ságvár: Képes fa and Bögöcsei-legelő, 19. 05. 2018, 3 females, 1 male, 02. 06. 2018, 1 female, 1 male; Somogybabod: Babodi-erdö, 02. 06. 2018, 1 female; Somogygeszti: municipal area, 13. 05. 2018, 1 male; Ságvár: Ali-rét, 19. 05. 2018, 4 males; Köröshegy: temető környéke, 13. 07. 2009, 1 female; Nikla: belterület, 14. 07. 2012, 1 female; Nagyberény: meadow at railway station, 28. 07. 2012, 1 female; Balatonlelle: Kis-hegy, 12. 07. 2012, 1 male.; Kereki: Homokfelvetés North, 08. 06. 2015, 1 male; Kereki: Lake, 29. 06. 2012, 1 male. Common species. Hostplant: Crataegus oxycantha.

Arge ochropus (Gmelin, 1790): Balatonszemes: Egyenes, 11. 06. 2007, 1 female, 1 male; Kereki: régi homokbánya, 11. 06. 2007, 1 male; Torvaj: belterület, 28. 07. 2012, 1 female. Pest of Rosa spp. Locally frequent.

Arge ustulata (Linnaeus, 1758): Somogygeszti: municipal area, 22. 04. 2018, 1 male. Sporadic. Larva on Salix alba vitellina, Salix alba, Salix aurita, Salix fragilis, Salix caprea, Betula pendula, Betula pubescens and Crataegus spp.

\section{Cimbicidae}

Corynis crassicornis (Rossi, 1790): Mezőcsokonya: temető körny., 19. 05. 2007, 1 female; Balatonboglár: Vár-hegy, 11. 05. 2010, 1 male. Sporadic. Hosts are Sedum spp. (S. album, S. acre and S. sexangulare).

\section{Cephidae}

Calameuta (Calameuta) filiformis (Eversmann, 1847): Kapoly: Kis-Koppány valley, 06. 05. 2018, 1 female, 19. 05. 2018, 1 female, 3 males; Somogybabod: Babodi-erdő, 13. 05. 2018, 1 female; Bonnya: Bonnyai-erdő. 12. 05. 2018, 1 female. Generally common species. Larva lives in stems of Arrhenaterum elatius, Phalaris arundinacea, Calamagrostis epigeios, Elytrigia repens and Phragmites communis. 
Calameuta (Calameuta) haemorrhoidalis (Fabricius, 1781): Ságvár: Ali-rét, 25. 04. 2018, 1 male; Mezőcsokonya: Alsónyíres: Csárdahely, 27. 04. 2018, 1 male; Kapoly: Kis-Koppány valley, 06. 05. 2018, 1 male; Ságvár: Képes fa and Bögöcsei-legelő, 06. 05. 2018, 1 male. Frequent species. Hostplant unknown.

Calameuta (Calameuta) pallipes (Klug, 1803): Somogyvár: Kupavári-erdő, 23. 04. 2018, 1 male, 29. 04. 2018, 1 male; Bábonymegyer: Patkó sír, 28. 04. 2018, 1 male; Magyaregres: Varga-bonyi árok, 27. 04. 2018, 1 male; Somogybabod: Somogybabodierdő, 28. 04. 2018, 1 female; Látrány, 24. 05. 2012, 1 female. Frequent species. Hostplants: diverse Poaceae.

Calameuta (Calameuta) punctata (Klug, 1803): Kapoly: Kis-Koppány valley, 30. 04. 2018, 1 female; Libickozma: belter., 20. 04. 2008, 1 female. Sporadic in Hungary.

Cephus brachycercus C. G. Thomson, 1871: Mezőcsokonya: Alsónyíres: Csárdahely, 27. 04. 2018, 1 female; Kapoly: Kis-Koppány valley, 30. 04. 2018, 1 female; Somogydöröcske: döröcskei-rét, 24. 04. 2018, 1 male; Ságvár: Ali-rét., 25. 04. 2018, 1 male. Widely distributed, sporadic species. Hostplant unknown.

Cephus nigrinus C. G. Thomson, 1871: Kapoly: Kis-Koppány valley, 26. 04. 20184 males, 1 female, 24. 04. 2018, 1 male; Somogygeszti: municipal area, 27. 04. 2018, 1 female; Hetes: meadow next to the cementery, 27. 04. 2018, 1 female; Ságvár: Ali-rét., 06. 05. 2018, 1 female. Frequent species. Hostplants: Milium effusum and Poa pratensis.

Cephus pygmeus (Linné, 1767): Bábonymegyer: Patkó sír, 28. 04. 2018, 2 males, 1 female; Magyaregres: Varga-bonyi árok, 29. 04. 2018, 1 male; 06. 05. 2018, 4 males, 6 females, 12. 0. 2018, 1 female, 27. 04. 2018, 2 females, 29. 04. 2018, 1 female; Kapoly: Kis-Koppány valley, 06. 05. 2018, 2 males, 2 females; 12. 05. 2018, 1 male, 15 females, 19. 05. 2018, 1 male, 3 females; Bonnya: Bonnyai-erdő, 1 male, 3 females; Ságvár: Alirét, 06. 05. 2018, 1 female, 25. 04. 2018, 1 female; Ságvár: Képes fa and Bögöcseilegelö, 28. 04. 2018, 1 female; Somogygeszti: municipal area, 13. 05. 2018, 1 female; Büssü: belter, 16. 05. 2009, 1 female. Common. Pest of cereals and grasses.

Cephus spinipes (Panzer, 1800) (syn. Cephus cultratus Eversmann, 1847): Ságvár: Ali-rét., 06. 05. 2018, 1 male, 19. 05. 2018, 1 male, 2 females; Polány: Gamási-árok, 21. 05. 2007, 1 female. Frequent species. Known hostplant: Phleum pratense.

\section{Tenthredinidae}

\section{Dolerinae}

Dolerus (Dicrodolerus) vestigialis (Klug, 1818): Kapoly: Kis-Koppány valley, 30.04. 2018, 1 male; Somogyfajsz: belter., 20. 04. 2008, 1 female. Common. Hostplants: Equisetum palustre, E. sylvaticum, E. arvense and E. pratense.

Dolerus (Dolerus) germanicus (Fabricius, 1775): Kapoly: Kis-Koppány valley, 06. 05. 2018, 1 female, 24. 04. 2018, 2 males, 26. 04. 2018, 2 males, 30. 04. 2018, 2 males; Balatonendréd: láprét, 16. 04. 2018, 1 female, 1 male; Karád, belter, 2008 07. 19, 1 female, Somogyaszaló: Novella, 23. 07. 2007. 1 female; Balatonföldvár: Kerék-rét, 13. 07. 2009, 1 female. Common. Larva on Equisetum arvense and E. palustre.

Dolerus (Oncodolerus) eversmanni W. F. Kirby, 1882: Balatonendréd: láprét, 16. 04. 2018, 1 female; Kapoly: Kis-Koppány valley, 24. 04. 2018, 1 female; Bábonymegyer: Kis-Koppány-valley, 16. 04. 2018, 1 male. Frequent. Larva on Equisetum arvense and E. palustre.

Dolerus (Poodolerus) aeneus Hartig, 1837: Igal: Andocsi úti-erdő, 14. 04. 2018, 1 male; Balatonendréd: láprét, 16. 04. 2018, 1 female; Ságvár: Képes fa and Bögöcseilegelö, 06. 05. 2018, 1 female; Pamuk: belter, 11. 04. 2009, 1 female. Sporadic. Hostplants: Graminae. 
Dolerus (Poodolerus) asper Zaddach, 1859: Buzsák: Kund-puszta, 02. 04. 2007, 1 male. Sporadic. Hostplants: Graminae and Cyperaceae.

Dolerus (Poodolerus) brevicornis Zaddach, 1859: Kisbárapáti: láprét, 14. 04. 2018, 1 female. Sporadic. Hostplant: Carex spp.

Dolerus (Poodolerus) gonager (Fabricius, 1781): Látrány: Öreg-hegy, 01. 04. 2008, 1 female. Generally common. Larva on Poaceae.

Dolerus (Poodolerus) haematodes (Schrank, 1781): Balatonendréd: láprét, 18. 04. 2018, 1 male. Frequent. Larva on Juncus, Scirpus, Crex and Gramineae.

Dolerus (Poodolerus) nigratus (O. F. Müller, 1776): Bábonymegyer: Kis-Koppányvalley, 16. 04. 2018, 1 female; Pusztaszemes: Szemesi-erdő, 23. 04. 2018, 1 female; Bonnya: Bonnyai-erdő, 24. 04. 2018, 1 female; Somogybabod: Somogybabodi-erdő, 25. 04. 2018, 1 female; Szólád: Nezde-puszta, 25. 04. 2018, 1 female; Mezőcsokonya: Alsónyíres: Csárdahely, 27. 04. 2018, 1 female; Kisbárapáti: láprét, 14. 04. 2018, 1 male; Bonnya-puszta, 08. 04. 2018, 1 male; Fiad: Fiadi-erdő, 14. 04. 2018; Szorosad: Koppánypatak, 08. 04. 2018, 1 male; Somogytúr: Túri-erdő, 15. 04. 2018, 1 male; Somogyfajsz: belter., 20. 04. 2008, 1 male. Common. Larva on Graminae including cereals.

Dolerus (Poodolerus) picipes (Klug, 1818): Kapoly: Kis-Koppány völgye, 24. 04. 2018, 1 female; Ádánd: Fehér-hegy, 17. 04. 2007, 1 female. Frequent. Larva on Graminae.

Dolerus (Poodolerus) puncticollis C. G. Thomson, 1871: Bábonymegyer: KisKoppány völgye, 16. 04. 2018, 1 female; Balatonendréd: láprét, 13. 04. 2018, 1 female; Kapoly: Kis-Koppány völgye, 08. 04. 2018, 1 female, 13. 04. 2018, 2 females; Bonnyapuszta, 08. 04. 2018, 1 female; Somogytúr: Túri-erdö, 15. 04. 2018, 1 female; Igal: Andocsi úti-erdő, 14. 04. 2018, 1 male; Nagybajom, 05. 04. 1963, 1 female; Vállus: Csetényi-rét, 26. 06. 2002., 1 male. Common. Larva on Graminae including cereals.

Dolerus (Poodolerus) quadrinotatus (Bíró, 1884): Tab: Koppány-valley, 08. 04. 2018, 1 female. Rare. Hostplant unknown.

Dolerus (Poodolerus) sanguinicollis (Klug, 1818): Ságvár: Ali-rét, 12. 05. 2018, 1 female. Sporadic.

\section{Selandrinae}

Birka (Birka) annulitarsis (Thomson, 1870): Pamuk: belter.: 11. 04. 2009, 1 female. Rare. Hostplant: Rubus spp.

Birka (Birka) cinereipes (Klug, 1816): Somogybabod: Somogybabodi-erdö, 22. 04. 2018, 2 females, 15. 04. 2018, 1 male. Sporadic. Hostplants: Myosotis spp.

Nesoselandria morio (Fabricius, 1781): Somogysárd: halastavak a régi temetőnél, 13. 05. 2018, 1 female. Frequent. Hostplants: Brachytecium reflexum, Ceratodon purpureus, Chenopodium album, Dicranum scoparium, Fragaria vesca, Hedwigia ciliata, Myosotis arvensis, Plagiomnium cuspidatum, Plagiothecium denticulatum, Polygonum aviculare, Polytrichum commune, Pseudobryum cinclidiodes, Sanionia uncinata, Stellaria media, Veronica chamaedrys and $V$. officinalis.

Selandria serva (Fabricius, 1793): Mezőcsokonya: Alsónyíres: Csárdahely, 27. 04. 2018, 1 male; Nágocs: belter, 25. 08. 2007, 1 female. Frequent. Host plants: Poaceae, Carex spp. and Juncus spp.

\section{Allantinae}

Allantus (Emphytus) cinctus (Linné, 1758): Bonnya: Bonnyai-erdő. 24. 04. 2018, 1 female; Kisbárapáti: belter.: 06. 08. 2008, 1 female; Csombárd: belterület, 07. 05. 2015, 1 male; Mernye: belterület, 10. 05. 2011, 1 female. Frequent. Hostplants: Rosa spp.

Allantus (Emphytus) melanarius (Klug, 1818): Vése, Csöpröndi road, 27. 04. 2012, 1 
female; Balatonboglár: Tabán, 11. 05. 2010, 1 female. Frequent. Hostplant: Cornus sanguinea.

Ametastegia (Protemphytus) carpini (Hartig, 1837): Patalom: Patalomi-erdő, 26. 04. 2018, 1 female. Sporadic. Hostplant: Geranium spp.

Ametastegia (Ametastegia) equiseti (Fallén, 1808): Ságvár: Ali-rét, 06. 05. 2018, 1 male. Frequent. Larva on Chenopodium album, Lythrum salicaria, Polygonum persicaria and Rumex acetosella.

Ametastegia (Protemphytus) pallipes (Spinola, 1808): Balatonendréd: láprét, 16. 04. 2018, 1 females; Szólád: Nezde puszta, 25. 04. 2018, 1 female. Frequent. Larva on Viola spp.

Ametastegia (Protemphytus) tenera (Fallén, 1808): Somogyaszaló: Deseda, 24. 04. 2018, 1 male, 26. 04. 2018, 2 males. Frequent. Larva on Rumex spp.

Athalia bicolor Serville, 1823: Kapoly: Kis-Koppány völgye, 26. 04. 2018, 1 female, 06. 05. 2018, 1 male, 12. 05. 2018, 2 males, 19. 05. 2018, 2 males; Somogysárd: halastavak a régi temetőnél, 29. 04. 2018, 1 female; Ságvár: Ali-rét, 06. 05. 2018, 1 female, 12. 05. 2018, 1 male; Bonnya: bonnyai-erdő, 12. 05. 2018, 1 female; Magyaregres: Varga-bonyi árok, 12. 05. 2018, 1 female, 20. 05. 2018, 1 female; Ságvár: Képes fa and Bögöcsei-legelö, 06. 05. 2018, 1 male; Balatonendréd: Endrédi-dombok, 06. 05. 2018, 1 male. Frequent. Hostplant unknown.

Athalia circularis (Klug, 1815): Somogyaszaló: Deseda: 24. 04. 2018, 4 males; Somogybabod: Somogybabodi-erdő, 28. 04. 2018, 3 males; Ecseny: belter, 14. 08. 2006, 1 male; Mezőcsokonya: temetö körny., 19. 05. 2007, 1 male; Torvaj: belterület, 28. 07. 2012, 1 male. Frequent. Hostplants: Arctium lappa, Ajuga reptans, Veronica beccabunga, V. longifolia, V. officinalis, Alliaria petiolata, Glechoma hederacea, Melampyrum, Capsella and Lycopus spp.

Athalia cordata Serville, 1823: Ságvár: Képes fa and Bögöcsei-legelö, 16. 04. 2018, 1 female; Mernyeszentmiklós: Szentmiklósi-erdő, 22. 04. 2018, 1 female; Somogydöröcske: döröcskei-rét, 24. 04. 2018, 1 female; Somogysárd: Halastavak, régi temető, 29. 04. 2018, 1 female; Somogyvár: Kupavári-erdő, 29. 04. 2018, 1 female, 2 males; Kapoly: Kis-Koppány völgye, 30. 04. 2018, 2 females, 2 males; Fiad: Kölesdpusztai-erdő, 24. 04. 2018, 1 male; Szólád: Nezde puszta, 25. 04. 2018, 1 male; Hetes: rét, a temetőnél, 27. 04. 2018, 1 male; Ságvár: Ali-rét, 28. 04. 2018, 1 male, 06. 05. 2018, 1 male; Somogyaszaló: Deseda, 02. 04. 2007, 1 female; Polány: Szentmiklósi-erdő, 26. 04. 2008, 1 male. Common. Larva on Misopates orontinum, Antirrhinum majus, Ajuga reptans, Teucrium scorodonia and Plantago spp.

Athalia rosae (Linné, 1758): Somogybabod: Somogybabodi-erdő , 22. 04. 2018, 1 female, 20. 05. 2018, 1 female; Hetes: meadow next to the cementery, 27. 04. 2018, 8 females, 3 males; Kapoly: Kis-Koppány völgye, 11. 08. 2018, 9 females, 5 males; Somogyaszaló: Novella, 23. 07. 2007. 1 male; Polány: Gamási-árok, 21. 05. 2007, 1 female, Somogygeszti: belter., 21. 05. 2007, 1 male. Common pest. Hostplants: Raphanus sativus, $R$. raphanistrum, Sinapis arvensis, Sisymbrium officinale, Armoracia rusticana, Barbarea sp., Brassica napus, B. juncea, B. rapa, B. oleracea, Tropaeolum majus, Sinapis arvensis, Alliara petiolata and Cardamine spp.

Empria hungarica (Konow, 1895): Ádánd: Fehér-hegy, 17. 04. 2007, 1 male. Rare. Hostplant unkown.

Empria liturata (Gmelin, 1790): Somogytúr: Túri-erdő, 15. 04. 2018, 1 female; Ságvár: Ali-rét, 25.04. 2018,1 female; Somogybabod: Somogybabodi-erdő, 15. 04. 2018, 1 male. Frequent. Hostplants: Fragaria and Geum spp.

Empria parvula (Konow, 1892): Balatonendréd: láprét, 13. 04. 2018, 1 male; Somogyacsa: Acsai-erdő, 15. 04. 2018, 1 male; Igal: Andocsi úti erdő, 14. 04. 2018, 1 male; Igal: Kavaljai-erdő. 05. 04. 2012, 1 female. Sporadic. 
Empria sexpunctata (Serville, 1823) (syn.: Empria klugii (Stephens, 1835)): Balatonendréd:láprét, 29. 04. 2018, 1 female, 16. 04. 2018, 1 male; Balatonendréd: Endrédi-dombok, 13. 04. 2018, 1 female, 16. 04. 2018, 1 male; Patalom: Patalomi-erdő, 26. 04. 2018, 1 female; Kapoly: Kis-Koppány völgye, 26. 04. 2018, 1 female; Bonnya: Bonnyai-erdő, 24. 04. 2018, 1 female; Somogyvár: Kupavári-erdő, 29. 04. 2018, 1 female, 23. 04. 2018, 1 male; Kereki: Vár-hegy, 23. 04. 2018, 1 female; Somogybabod: Somogybabodi-erdő, 22. 04. 2018, 1 female, 2 males, 15. 04. 2018, 1 male; Ságvár: Alirét, 25. 04. 2018, 1 female; Fiad: Kölesdpusztai-erdö, 24. 04. 2018,1 male; Somogyaszaló: Deseda, 15. 04. 2018, 1 male; Mezőcsokonya: Csokonyai-erdő, 25. 04. 2018, 1 male; Kisbárapáti: láprét, 14. 04. 2018, 1 male; Fiad: Fiadi-erdő, 14. 04. 2018, 1 male, Törökkoppány: Koppányi-erdő, 08. 04. 2018, 1 male. Frequent. Larva on Geum spp.

Empria tridens (Konow, 1896): Somogyaszaló: Deseda, 15. 04. 2018, 1 female, 1 male; Ságvár: Ali-rét, 25. 04. 2018, 1 female; Igal: Andocsi úti erdő, 14. 04. 2018, 1 male; Kereki: Vár-hegy, 14. 04. 2018, 1 male. Frequent. Hostplants: Geum spp. and Rubus idaeus.

Eriocampa ovata (Linné, 1761): Libickozma: belter., 07. 07. 2007, 1 female. Frequent on Alnus glutinosa and A. incana.

Harpiphorus lepidus (Klug, 1818): Somogysárd: halastavak a régi temetőnél, 25. 04. 2018, 1 female. Rare. Hostplants: Quercus spp.

Monostegia abdominalis (Fabricius, 1798): Magyaregres: Gombás parkerdő, 26. 04. 2012, 1 female. Sporadic. Hostplants: Lysimachia, Anagallis, Glaux maritima.

\section{Heterarthrinae}

Caliroa cinxia (Klug, 1816): Hetes: belter., 19. 05. 2007, 1 male. Sporadic. Larva on Quercus spp.

Heterarthrus vagans (Fallén, 1808): Magyaregres: Varga-bonyi árok, 20. 05. 2018, 1 larva in mine. Sporadic. Hostplants: Alnus spp.

Hinatara nigripes (Konow, 1907): Kereki: Vár-hegy, 14. 04. 2018, 1 female. Rare. Hostplant: Acer capestre.

\section{Blennocampinae}

Claremontia alternipes (Klug, 1816): Magyaregres: Varga-bonyi árok, 15. 04. 2018, 1 female; Bonnya: Bonnyai-erdő. 24. 04. 2018, 1 female; Somogyaszaló: Deseda, 15. 04. 2018, 1 male; Somogyacsa: Acsai-erdő, 15. 04. 2018, 1 male; Somogyaszaló: Deseda, 18. 04. 2012, 1 female. Sporadic. Hostplant: Rubus idaeus.

Claremontia brevicornis (Brischke, 1883): Fiad: Kölesdpusztai-erdö, 24. 04. 2018, 1 female. Sporadic. Hostplants: Fragaria spp., Sanguisorba spp. and Potentilla reptans.

Claremontia puncticeps (Konow, 1886): Ságvár: Képes fa and Bögöcsei-legelö, 13. 04. 2018, 1 female; Pusztaszemes: Szemesi-erdő, 14. 04. 2018, 1 female. Sporadic. Hostplants: Fragaria spp., Sanguisorba spp. and Potentilla reptans.

Claremontia waldheimii (Gimmerthal, 1847): Igal: Andocsi úti-erdő, 14. 04. 2018, 1 female. Frequent. Hostplant: Geum urbanum.

Eutomostethus ephippium (Panzer, 1798): Balatonendréd: láprét, 16. 04. 2018, 1 female, 1 male, 29. 04. 2018, 1 male; Magyaregres: Varga-bonyi árok, 22. 04. 2018, 1 female, 1 male, 27. 04. 2018, 1 male; Somogysárd: Halastavak a régi temetőnél, 23. 04. 2018, 2 females; Somogybabod: Somogybabodi-erdő, 25. 04. 2018, 2 females, 2 males, 13. 05. 2018, 1 male; Somogyaszaló: Deseda, 26. 04. 2018, 1 female; Somogyvár: Kupavári-erdő, 29. 04. 2018, 3 females, 1 male, 23. 04. 2018, 3 males; Ságvár: Képes fa and Bögöcseilegelö, 16. 04. 2018, 1 male, 28. 04. 2018, 3 males; Kereki: Vár-hegy, 23. 04. 2018, 1 male; Kisbárapáti: láprét, 24. 04. 2018, 1 male; Bonnya: Bonnyai-erdő, 24. 04. 2018, 1 male; Somogymeggyes: Kis-Koppány völgye, 28. 04. 2018, 1 male; Szólád: Nezde puszta, 25. 


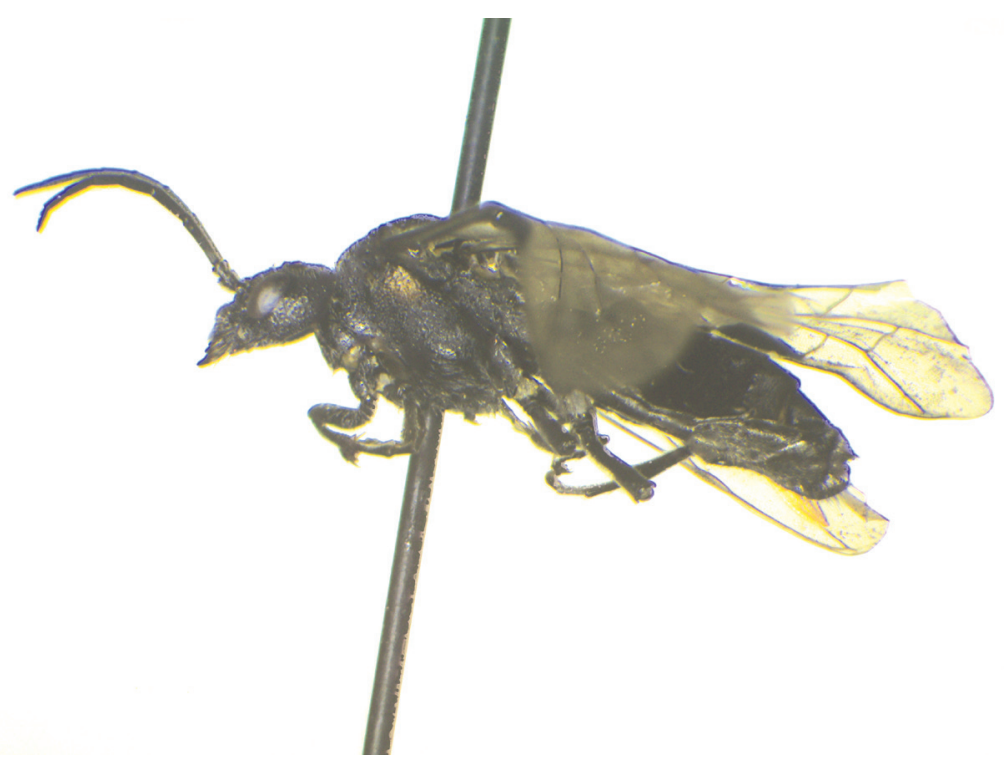

Fig. 5: Dolerus (Poodolerus) quadrinotatus (Bíró, 1884) new colour variation

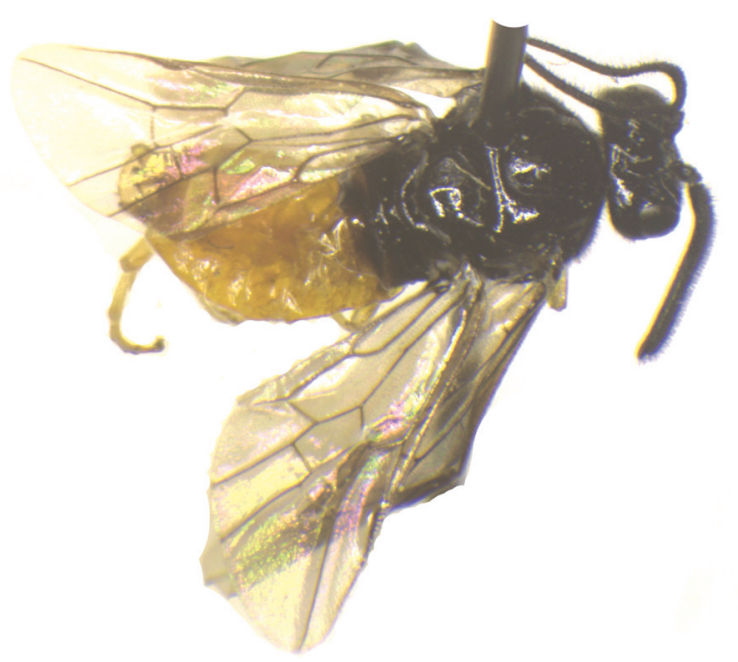

Fig. 6: Aprosthema austriacum (Konow, 1892) 
04. 2018, 1 male; Tengőd: Tengődi-erdő, 26. 04. 2018, 1 male; Hetes: rét a temető mellett, 27. 04. 2018, 1 male; Mezőcsokonya: Alsónyíres: Csárdahely, 27. 04. 2018, 1 male; Ságvár: Ali-rét, 28. 04. 2018, 1 male, 06. 05. 2018, 2 males, 12. 05. 2018, 1 male; Kisbárapáti: Márton-hegy, 07. 05. 2008, 1 male; Somogyaszaló: Deseda, 18. 04. 2012, 1 male; Somogyfajsz: Vadászház, 14. 07. 2012, 1 male. Common species. Larva on Graminae.

Eutomostethus gagathinus (Klug, 1816): Somogybabod: Somogybabodi-erdö, 22. 04. 2018, 1 male. Sporadic.

Halidamia affinis (Fallén, 1807): Balatonendréd: láprét, 16. 04. 2016, 1 female; Somogyvár: Kupavári-erdő, 23. 04. 2018, 1 female; Magyaregres: Varga-bonyi árok, 15. 04. 2018, 1 female; Balatonlelle: Kis-hegy, 01. 04. 2008, 1 female; Polány: Szentmiklósi erdö, 26. 04. 2008, 1 female. Frequent. Hostplants: Galium aparine and G. molugo.

Monophadnoides rubi (Harris, 1845) (syn.: Monophadnoides geniculatus (Hartig, 1837)): Kapoly: Kis-Koppány völgye, 13. 04. 2018, 1 male. Frequent. Hostplant: Rubus fructicosus.

Monophadnoides ruficruris (Brulle, 1832): Somogyaszaló: Deseda, 18. 04. 2012, 1 female. Frequent. Hostplant: Rubus idaeus.

Monophadnus longicornis (Hartig, 1837, sensu Zombori, 1990): Kisbárapáti: láprét, 24. 04. 2018, 3 males. Sporadic. Hostplant unknown. (Well known species, generally attributed to Hartig, however not identical with M. longicornis Hartig).

Monophadnus monticola (Hartig, 1837): Somogyaszaló: Deseda, 25. 04. 2012, 1 female. Frequent. Larva on Helleborus spp. and Ranunculus acris.

Monophadnus pallescens (Gmelin, 1790): Somogysárd: Halastavak a régi temetőnél, 23. 04. 2018, 1 female; Kapoly: Kis-Koppány völgye, 24. 04. 2018, 1 female; Somogybabod: Somogybabodi-erdö, 15. 04. 2018, 1 female; Kereki: Vár-hegy, 14. 04. 2018, 1 female; Fiad: Vadas-erdő, 05. 04. 2009, 1 female. Common. Hostplants: Ranunculus acris, $R$. repens, $R$. lanuginosus and Anemone nemorosa.

Monphadnus spinolae (Klug, 1816): Bonnyapuszta, 30. 06. 2009, 1 male; Gamás: Kisbári út, 06. 05. 2010, 1 male. Sporadic. Hostplants: Clematis vitalba and C. flammula. Pareophora pruni (Linnaeus, 1758): Ságvár: Ali-rét, 25. 04. 2018. Frequent. Larva on Prunus spinosa.

Phymatocera aterrima (Klug, 1816): Somogyvár: Kupavári-erdő, 23. 04. 2018, 1 male. Frequent. Hostplants: Polygonatum spp.

Stethomostus fuliginosus (Schrank, 1781): Polány: Gamási-árok, 21. 05. 2007, 1 male.

Frequent. Larva on Ranunculus acris, $R$. repens and $R$. sceleratus.

\section{Tenthredininae}

Aglaostigma (Astochus) aucupariae (Klug, 1817): Somogytúr: Hárshegyi vadászház, 22. 04. 2018, 1 female; Somogydöröcske: Döröcskei-rét, 24. 04. 2018, 1 female; Tengőd: Tengődi-erdő, 26. 04. 2018, 1 female; Igal: Andocsi úti erdő, 14. 04. 2018, 1 female; Ságvár: Képes fa and Bögöcsei-legelö, 16. 04. 2018, 1 male; Kisbárapáti: láprét, 14. 04. 2018, 1 male; Kapoly: Kis-Koppány völgye, 08. 04. 2018, 1 male; Ságvár: Képes fa and Bögöcsei-legelö, 13. 04. 2018, 2 males; Fiad: Vadas-erdő, 05. 04. 2009, 1 male, Siófok: Töreki, 12. 03. 2007, 1 female, Igal: belter.: 19. 04. 2008, 1 female; Mernye: Amerikai kereszt, 31. 03. 2012, 1 male; Somogybabod: Szent-kút, 31. 03. 2011, 1 male; Visz: Karádi út mentén, 31. 03. 2011, 1 female; Somogyaszaló: Deseda, 04. 04. 2011, 1 female.. Common. Larva on Galium mollugo and G. boreale.

Aglaostigma (Astochus) fulvipes (Scopoli, 1763): Kaposfüred: Kaposfüredi út, 22. 04. 2018, 1 female; Somogygeszti: municipal area, meadow at Széchenyi street, 27. 04. 2018, 4 females, 22. 04. 2018, 1 female; Magyaregres: Varga-bonyi árok, 27. 04. 2018, 1 female; Ságvár: Képes fa and Bögöcsei-legelö, 28. 04. 2018, 1 female; Somogybabod: 
Somogybabodi-erdő, 22. 04. 2018, 1 female, 25. 04. 2018, 1 female; Mernyeszentmiklós: Szentmiklósi-erdő, 22. 04. 2018, 1 female; Kapoly: Kis-Koppány völgye, 24. 04. 2018, 1 female, 30. 04. 2018, 1 male; Igal: Andocsi úti erdő, 14. 04. 2018, 3 males, 16. 04. 2018, 2 males; Látrány: Öreg-hegy, 01. 04. 2008, 1 male, Polány: Szentmiklósi-erdö, 26. 04. 2008, 1 male; Magyaregres: Deseda, arborétum, 20. 04. 2011, 1 male; Somogyaszaló: Deseda, 25. 04. 2012, 1 male. Common. Larva on Galium mollugo and G. verum.

Macrophya (Macrophya) albicincta (Schrank, 1776): Somogysárd: Halastavak, 23. 04. 2018, 2 females, 29. 04. 2018, 1 male; Somogytúr: Hárshegyi vadászház, 22. 04. 2018, 1 female, 1 male; Somogyaszaló: Deseda, 26. 04. 2018, 1 female, 1 male; Pusztaszemes: Szemesi-erdő, 23. 04. 2018, 2 females, 14. 04. 2018, 1 female; Ságvár: Képes fa and Bögöcsei-legelö, 60. 05. 2018, 1 female, 13. 04. 2018, 1 female, 1 male; Somogyacsa: Acsai-erdő, 15. 04. 2018, 2 females; Kapoly: Kis-Koppány völgye, 26. 04. 2018, 1 female, 30. 04. 2018, 1 female; Mezőcsokonya: Alsónyíres: Csárdahely, 27. 04. 2018, 1 female, 1 male; Somogymeggyes: Kis Koppány-völgye, 28. 04. 2018, 1 female; Magyaregres: Varga-bonyi árok, 29. 04. 2018, 1 female; Igal: Andocsi úti-erdő, 14. 04. 2018, 1 female; Kára, 13. 04. 2018,1 female, 1 male; Bonnya: Bonnyai-erdő, 24. 04. 2018, 2 males; Ságvár: Ali-rét, 25. 04. 2018, 1 male, 28. 04. 2019, 1 male; Somogygeszti: belter., 21. 05. 2007, 1 female; Somogyaszaló: Deseda, 02. 04. 2007, 1 female, 25. 04. 2012, 1 female; Balatonszemes: Bagó-domb, 07. 04. 2009, 1 female; Kaposvár: Tókajtó, 01. 05. 2009, 1 female; Somogyvár: Brézai-erdő, 11. 04. 2009, 1 male; Polány: Szentmiklósi-erdő, 26. 04. 2008, 1 female; Balatonlelle: Kis-hegy, 18. 04. 2011, 1 female; Töröcske: belterület, 06. 05. 2015, 1 female; Balatonboglár: Vár-hegy, 03. 04. 2012, 1 female. Common. Hostplants: Sambucus ebulus, S. nigra, S. racemosa, Valeriana officinalis and Viburnum opalus.

Macrophya (Macrophya) annulata (Geoffroy, 1785): Balatonendréd: Endrédi-dombok, 06. 05. 2018, 1 male. Frequent. Larva on Potentilla reptans, Origanum vulgare, Euphorbia, Rosa, Rubus and Sambucus spp.

Macrophya (Macrophya) chrysura (Klug, 1817): Mezőcsokonya: temető körny., 19.. 05. 2007, 1 male. Sporadic. Hostplant: Daucus carota.

Macrophya (Macrophya) crassula (Klug, 1817): Nikla: belterület, 01. 08. 2015, 1 female. Sporadic. Hostplant: Sambucus ebulus.

Macrophya (Macrophya) duodecimpunctata (Linné, 1758): Kapoly: Kis-Koppány völgye, 30. 04. 2018, 1 male; Polány: Gamási-árok, 21. 05. 2007, 1 female; Somogyaszaló: Deseda, 18. 04. 2012, 1 male. Common. Hostplants: Graminae, Cyperaceae and Carex spp.

Macrophya (Macrophya) diversipes (Schrank, 1782): Somogyaszaló: Novella, 30. 07. 2007, 1 male. Sporadic. Hostplant unknown.

Macrophya (Macrophya) montana (Scopoli, 1763): Somogybabod: Somogybabodierdő, 12. 05. 2018, 1 female, 02. 06. 2018, 6 females, 2 males, 20. 05. 2018, 3 females, 2 males; Ságvár: Ali-rét, 12. 05. 2018, 9 females, 6 males, 19. 05. 2018, 4 males; Kapoly: Kis-Koppány völgye, 12. 05. 2018, 1 female, 1 male, 19. 05. 2018, 2 females; Ságvár: Képes fa and Bögöcsei-legelö, 02. 06. 2018, 1 female, 1 male, 19. 05. 2018, 3 males; Magyaregres: Varga-bonyi árok, 20. 05. 2018, 1 female; Igal: Andocsi úti-erdő, 12. 05. 2018,1 male; Bonnya: Bonnyai-erdő, 12. 05. 2018, 1 female, 2 males; Polány: Gamásiárok, 21. 05. 2007, 1 male; Somogyaszaló: belter, 21. 05. 2007, 1 female; Büssü: belter., 16. 05. 2009, 1 female. Common. Hostplant: Rubus caesius.

Macrophya (Macrophya) postica (Brullé, 1832): Bonnya: belter., 08. 06. 2009, 1 male. Frequent. Hostplant unknown.

Macrophya (Macrophya) rufipes (Linné, 1758): Somogyaszaló: Novella, 30. 06. 2007, 1 female. Sporadic. Larva on Agrimonia eupatoria. 
Pachyprotasis rapae (Linné, 1767): Somogygeszti: municipal area, 27. 04. 2018, 1 female, 22. 04. 2018, 1 male; Somogybabod: Somogybabodi-erdő, 25. 04. 2018, 4 males, 28. 04. 2018, 2 males; Kapoly: Kis-Koppány völgye, 06. 05. 2018, 1 male; Ecseny: belter, 14. 08. 2006, 1 female; Kisbárapáti: belter., 06. 08. 2008, 1 female. Common. Hostplants: Solanum tuberosum, Pedicularis palustris, Angelica sylvestris, Veronica beccabunga, Betonica officinalis, Corylus avellana, Salix caprea, Fraxinus excelsior, Tussilago farfara, Symphoricarpos albus, Scrophularia, Solidago, Verbascum, Origanum, Atropa, Sarothamnus, Senecio, Polygonum, Aspidium, Epilobium, Hypericum, Galeopsis, Mentha, Polystichum, Plantago, Quercus and Stachys spp.

Rhogogaster (Rhogogaster) chlorosoma (Benson, 1943): Ecseny: belter., 28. 06. 2007, 1 female. Generally frequent. Hostplants: Pteridium aquilinum, Alnus glutinosa, Circaea, Prunus spp., Ranunculus spp., Rosa spp., Salix alba, S. purpurea, Stellaria spp., Filipendula ulmaria, Populus tremula, Padus spp., Betula spp., Corylus avellana and Sorbus spp.

Sciapteryx consobrina (Klug, 1816): Magyaregres: Varga-bonyi árok, 27. 04. 2018, 2 females; Somogygeszti: municipal area, 27. 04. 2018, 1 female, 22. 04. 2018, 1 female; Ságvár: Ali-rét, 28. 04. 2018, 2 females; Ságvár: Képes fa and Bögöcsei-legelö, 28. 04. 2018, 3 females, 16. 04. 2018, 2 males, 08. 04. 2018, 1 male; Somogysárd: Halastavak, 25. 04. 2018, 1 female; Somogybabod: Somogybabodi-erdö, 25. 04. 2018, 1 female, 15. 04. 2018, 1 female, 22. 04. 2018, 2 females; Ságvár: Ali-rét, 25. 04. 2018, 1 female; Törökkoppány: Koppányi-erdő, 08. 04. 2018, 1 male; Gamás: Vadépuszta, 03. 04. 2007, 1 female; Somogyaszaló: Deseda, 25. 04. 2012, 1 female; Igal: Kavaljai-erdő, 05. 04. 2012, 1 male. Common. Larval hosts: Adoxa spp., Anemone spp. and Ranunculus ficaria.

Sciapteryx costalis (Fabricius, 1775): Balatonendréd: Endrédi-dombok, 13. 04. 2018, 1 male; Kapoly: Kis-Koppány völgye, 13. 04. 2018, 1 male. Frequent. Hostplant: Ranunculuis acris.

Tenthredo (Zonuledo) amoena Gravenhorst, 1807: Kisbárapáti: Márton-hegy, 06. 08. 2008, 1 female. Sporadic. Hostplants: Hypericum perforatum and H. maculatum.

Tenthredo (Cephaledo) bifasciata ssp. violacea (Ed. André, 1881): Ságvár: Képes fa and Bögöcsei-legelö, 19. 05. 2018, 1 male; Bábonmegyer: Patkó-sír, 06. 05. 2018, 1 female; Somogybabod: Somogybabodi-erdő, 02. 06. 2018, 1 female; Kereki: régi homokbánya, 11. 06. 2007, 1 female; Torvaj: belterület, 28. 07. 2012, 1 male; Ecseny: belterület, 03. 08. 2012, 1 female. Frequent. Hostplant unknown.

Tenthredo (Cephaledo) bifasciata rossii (Panzer, 1804): Tab: Lullai út, 20. 07. 2009, 1 male, Bonnya: belter., 08. 06. 2009, 1 male. Frequent. Hostplants: Scrophularia and Verbascum spp.

Tenthredo (Endotethryx) campestris Linné, 1758: Somogybabod: Somogybabodierdö, 20. 05. 2018, 1 female, 3 males; Somogygeszti: municipal area, 27. 04. 2018, 1 female. Frequent. Hostplant: Aegopodium podagraria.

Tenthredo (Eurogaster) mesomela Linné, 1758: Somogybabod: Somogybabodi-erdö, 20. 05. 2018, 1 female. Frequent. Larval hosts: Polygonum persicaria, Arctium lappa, Heracleum spp., Ranunculus spp., Epilobium spp., Rumex spp., Salix spp., Veronica sp., Tussilago spp., Petasites sp., Senecio sp., Solidago sp. and Stachys spp.

Tenthredo (Zonuledo) distinguenda (Stein, 1885): Ságvár: Képes fa and Bögöcseilegelö, 19. 05. 2018, 1 male. Sporadic. Hostplant unknown.

Tenthredo (Cephaledo) excellens (Konow, 1886): Balatonendréd: Endrédi-dombok, 16. 04. 2018, 1 female; Kapoly: Kis-Koppány völgye, 30. 04. 2018, 1 female. Sporadic. Hostplant unknown.

Tenthredo (Tenthredo) scrophulariae Linné, 1758: Kapoly: Kis-Koppány völgye, 06. 05. 2018, 1 female. Sporadic. Larva on Scrophularia and Verbascum spp. 
Tenthredo (Tenthredo) thompsoni (Curtis, 1839): Miklósi: belter., 25. 08. 2007, 1 female. Frequent. Hostplant unknown.

Tenthredo (Tenthredo) vespa Retzius, 1783: Alsóbogát: Fishing pond, 08. 08. 2012, 1 female. Frequent. Hostplants: Lonicera caprifolium, Syringa vulgaris, Viburnum opulus, Fraxinus excelsior, Jasminum officinale, Rosa, Spiraea, Acer platanoides, Ligustrum vulgare, Symphoricarpos albus, S. alba.

Tenthredo (Zonuledo) zonula Klug, 1817: Magyaregres: Varga-bonyi árok, 20. 05. 2018, 1 female; Ságvár: Ali-rét, 19. 05. 2018, 2 females; Bábonymegyer: Patkó sír, 28. 04. 2018, 1 female; Ságvár: Képes fa and Bögöcsei-legelö, 19. 05. 2018, 1 male; Kereki: régi homokbánya, 11. 06. 2007, 1 female, Kercseliget: belter, 16. 05. 2009, 1 male; Bonnyapuszta 05. 05. 2012, 1 male; Gamás: Kisbári út, 06. 05. 2012, 1 female; Látrány, 24. 05. 2012, 1 female. Frequent. Hostplant: Hypericum perforatum.

Tenthredopsis nassata (Linné, 1767): Gamás: cementery, 07. 05. 2015, 1 female. Frequent. Hostplants: Dactylis glomerata, Deschampsia caespitosa, D. calmagrostis, Flexuosa spp., Holcus spp., Lolium perenne, Agropyron spp., Carex spp., Anthriscus silvestris and Artemisia spp.

Tenthredopsis sordida (Klug, 1817): Magyaregres: Varga-bonyi árok, 29. 04. 2018, 1 female; Kapoly: Kis-Koppány völgye, 19. 05. 2018, 1 female, 30. 04. 2018, 1 male; Hetes: kaszáló a temetőnél, 27. 04. 2017, 1 male; Somogymeggyes: Kis-Koppány völgye, 28. 04. 2018, 1 male; Somogysárd: Halastavak, 29. 04. 2018, 1 male. Frequent. Larva on Arrhenatherum elatius, Lolium perene, Carex spp., Calamagrostis sp. and Dactylis glomerata.

Tenthredopsis stigma (Fabricius, 1798): Kapoly: Kis-Koppány völgye, 06. 05. 2018, 1 male. Generally frequent. Hostplant: Triticum intermedium.

\section{Nematinae}

Pachynematus (Larinematus) imperfectus (Zaddach, 1876) (curently Euura imperfectus (Zaddach, 1876): Somogytúr: Túri-erdö, 15. 04. 2018, 1 female. Rare. Hostplant: Laris spp.

Pachynematus (Pachynematus) vagus (Fabricius, 1781) (currently Euura vagus (Fabricius, 1781)?: Somogybabod: Somogybabodi-erdő, 25. 04. 2018, 1 female. Sporadic. Larva on Carex spp. Recently genus Pachynematus synonimised under Euura.

Cladius (Cladius) pectinicornis (Geoffroy, 1785): Kisbárapáti: láprét, 14. 04. 2018, 1 male; Kapoly: Kis Koppány-völgye, 26. 04. 2018, 1 female; Ságvár: Ali-rét, 28. 04. 2018, 1 female; Ecseny: belter, 06. 08. 2006, 1 male. Frequent. Larval hosts: Fragaria, Rosa, Filipendula spp., Poterium sanguisorba, Lamiastrum galeobdolon and Comarum palustre.

Cladius (Priophorus) brullei (Dahlbom, 1835): Kapoly: Kis Koppány-völgye, 26. 04. 2018, 1 female; Ságvár: Ali-rét, 12. 05. 2018, 1 female. Frequent. Larva on Rubus spp.

Hoplocampa flava (Linné, 1761): Balatonlelle: Kis-hegy, 01. 04. 2008, 1 male. Occasional insect pest. Larva on Prunus domestica and Prunus spinosa.

Hoplocampa fulvicornis (Panzer, 1801) (syn.: Hoplocampa rutilicornis (Klug, 1816)): Miklósi, 08. 04. 2018, 3 females, 1 male. Sporadic, locally frequent. Larva on Prunus spinosa.

Hoplocampa crataegi (Klug, 1816): Kapoly: Kis Koppány-völgye, 26. 04. 2018, 1 female. Frequent. Hostplants: Crataegus spp.

Mesoneura opaca (Fabricius, 1775): Somogysárd: Halastavak, 23. 04. 2018, 1 female. Sporadic. Larva on Quercus robur.

Nematus (Nematus) lucidus (Panzer, 1801): Somogygeszti: municipal area, 22. 04. 2018, 1 female; Balatonlelle: Kis-hegy, 01. 04. 2008, 1 male. Sporadic, locally frequent. Larva on Crataegus and Prunus spinosa. 
Nematus (Pteronidea) oligospilus Förster, 1854: Balatonendréd: láprét, 16. 04. 2018, 1 female. Frequent. Larva on Salix spp.

Nematus (Pteronidea) myosotidis (Fabricius, 1804): Somogysárd: Halastavak, 29. 04. 2018, 1 female; Balatonendréd: láprét, 16. 04. 2018, 1 female; Kapoly: Kis Koppányvölgye, 30. 04. 2018, 1 male; Bonnya: Bonnyai-erdő, 24. 04. 2018, 1 male; Kisbárapáti: láprét, 24. 04. 2018,1 male; Somogygeszti: municipal area, 22. 04. 2018,1 male; Bábonymegyer: legelő, 16. 04. 2018,1 male; Polány: halastó, 22. 04. 2018,1 male. Common. Larval hosts: Onobrychis and Trifolium spp.

Pontania (Pontania) proxima (Serville, 1823): Ságvár: Képes fa and Bögöcsei-legelö, 06. 05. 2018, 3 galls; Kapoly: Kis Koppány-völgye, 06. 05. 2018, 2 galls. Frequent, larva on Salix fragilis and S. alba.

Pristiphora (Pristiphora) armata (C. G. Thomson, 1863): Gamás: belter., 22. 08. 1999, 1 male, Ságvár: Jaba-völgy, 20. 04. 1998, 1 male. Frequent. Larva on Crataegus spp.

Pristiphora (Micronematus) monogyniae (Hartig, 1840): Igal: Andocsi úti-erdő, 14. 04. 2018, 1 female; Bonnya: Bonnyai-erdő, 24. 04. 2014, 1 female. Sporadic. Hostplant: Prunus spinosa ocasionally $P$. domestica.

Pristiphora subbifida (C. G. Thomson, 1871): Ságvár: Ali-rét, 25. 04. 2018, 1 female; Mernyeszentmiklós: Szentmiklósi-erdő, 22. 04. 2018; Somogytúr: Túri-erdő, 15. 04. 2018, 1 female. Sporadic. Hostplants: Acer campestre, sometimes A. pseudoplatanus and A. orientale.

\section{Results}

\section{Rare species}

Aprosthema austriacum (Konow, 1892). Fig. 6. Rare species, from Hungary we have specimens Budapest: Jánoshegy and Hársbokorhegy, from the Mecsek Hills and also from Szilvásvárad. From the other regions of the Carpathian Basin it is recorded from Lepsa (Romanian Carpaths) and there are also 2 indefinite records from Burgenland and Subcarpathia.

Dolerus (Poodolerus) quadrinotatus (Bíró, 1884). Fig. 5. Rare species, out of the Carpathian Basin it is known only from Spain (one specimen). From Hungary, we have specimens from Szeged: Újszeged, Simontornya, Látrány, Battonya: Tompapuszta and Sződ. From Slovakia, it is known from Viničky (Szöllőske) and from Transylvania from Peér, Tasnád, Borosjenő and Németbogsán. Our specimen is a new colour variation: the red dorsal spot is completely missing, only the lateral red spots are present.

Birka (Birka) annulitarsis (Thomson, 1870). Known occurrences from Hungary: Bakony, Mecsek Szilvásvárad, Aggtelek and Jósvafö. From the Carpathian Basin it is also known from Devín (Dévény), Javorina (Balázsvágás), Mošovce (Mosóc), Štefanová (Istvánkirályfalva) Zemianske Podhradie (Nemesváralja), Adamovské Kochanovce (Adamóckohanóc), Moravské Lieskové (Morvamogyoród) from Slovakia; Nagyszeben (Sibiu), Magura, Horaita Zeteváralja (Sub Cetatea): Szencsed patak, Homoródfürdő (Baile Homorod) from Romania; Haňkovice-údolie Latorice, Pop Ivan, Tiszabogdány, Brebenyeszkul (Bogdan) and Trebusafejérpatak (Dilove) from Subcarpathia.

Empria hungarica (Konow, 1895). We have data from Hungary, from Magyaralmás, Simontornya, Hármashatárhegy, Budakeszi, Ócsa, Jósvafö, Lébény and Köveskál. It is also known from Neusiedler See (Austria); Štúrovo (Párkány), Devínska Kobyla (Pozsony: Dévény) (Slovakia); Mehádia, Arad, Krassó-Szörény (Caras-Severin), 
Szenterzsébet (Gusterita), Zeteváralja (Sub Cetatea): Szencsed patak, Homoródfürdő (Baile Homorod) (Romania); Tiszabogdány(Bogdan) and Brebenyeszkul (Subcarpathia).

Harpiphorus lepidus (Klug, 1818). We have specimens and records from Szendehely: Katalin-puszta, Szentbalázs, Budapest, Badacsony, Nadap, Őrszentmiklós, Peszér, Nova. From Slovakia, it is known from Sitno (Szitnya), Malacky (Malacka), Ihelník, Kňaží vrch (Pap kő), Trenčianske Bohuslavice-Hájnica (Bogoszló: Hajnica), Skalka nad Váhom (Vágsziklás), Zemianske Podhradie (Nemesváralja) and Turecký vrch (Nové Mesto n.V.). Romanian records: Kereszténysziget (Cristian), Nagyszeben (Sibiu), Hadad (Hodod) and Homoródkeményfalva (Comanesti).

Hinatara nigripes (Konow, 1907). Hungarian occurence: Kóspallag, Szokolya, Verőce, Lébény, Nagykovácsi, Budapest and Aggtelek. Other records from the Carpathian Basin: Magura-Kisdisznód (Magura Cisnadioarei), Nagyszeben (Sibiu) (Transylvania). It is also recorded from the Czech Carpathians and Slavonia.

Pachynematus (Larinematus) imperfectus (Zaddach, 1876) (curently Euura imperfectus (Zaddach, 1876). This very rare species is known only from Kozí chrbát-Pol'ana Mts (Kecskehát) from Slovakia, Zirc from Hungary and Királymező (Ust-Tschorna) from Subcarpathia.

\section{Dominant species}

Cephus pygmeus (Linné, 1767) was collected in the highest number. Besides Cephus pygmeus (Linné, 1767), Macrophya albicincta (Schrank, 1776), Macrophya montana (Scopoli, 1763), Eutomostethus ephippium (Panzer, 1798) Aglaostigma fulvipes (Scopoli, 1763), Aglaostigma aucupariae (Klug, 1817), Sciapteryx consobrina (Klug, 1816) and Empria sexpunctata (Serville, 1823) were the most frequent species. These eigth species make $47 \%$ of the total material.

\section{References}

ACHTERBERG, C. 2013: Hymenoptera in Fauna Europaea version 2.6.2. http://www.faunaeur.org . last accessed $10^{\text {th }}$ August 2017.

Gyurkovics, H. \& Haris, A. 2012: Sawflies (Hymenoptera: Symphyta) from Szeged and its surroundings (SE Hungary) - Natura Somogyiensis 22: 163-182.

Haris, A. 1998: Somogy Megyei Múzeum levéldarázs-gyüjteménye (Hymenoptera: Symphyta) - Somogyi Múzeumok Közlemenyei 13: 275-285.

Haris, A. 2001: Somogy megye levéldarázs-alkatúinak katalógusa (Hymenoptera, Symphyta), Catalogue of the sawfly fauna of Somogy county (Hymenoptera: Symphyta) -Natura Somogyiensis 1 261-268.

Haris, A. 2003: Növényevő darazsak a Látrányi Puszta Természetvédelmi Területről (Hymenoptera: Symphyta) -Natura Somogyiensis 5 201-207.

Haris, A. 2009: Sawflies of the Zselic Hills, SW Hungary (Hymenoptera: Symphyta) - Natura Somogyiensis 15: 127-158.

Haris, A. 2011: Sawflies of the Börzsöny Mountains (North Hungary) (Hymenoptera: Symphyta) - Natura Somogyiensis 19: 149-176.

MocsáRY, S. 1900: Ordo Hymenoptera. p. 7-113. In: Paszlavsky, J. (ed.): Fauna Regni Hungariae, Regia Societas Scientiarum Naturalium Hungarica, Budapest.

MóczÁr, L., \& Zombori, L. 1973: Tenthredinoidea - Levéldarázs-alkatúak I. In: Fauna Hungariae, Akadémiai Kiadó, Budapest, 111, 11(2), 128 p.

Roller, L. 1993: New records of sawflies (Hymenoptera: Symphyta) from Slovakia. -Entomological Problems 24(2): 81-84.

Roller, L. 1994: Faunistics records. Symphyta. -Entomological Problems 25(2): 24. 
RoLler, L. 1996: New records of sawflies (Hymenoptera, Tenthredinidae) in Slovakia. -Biologia, Bratislava 51(1): 549-550.

Roller, L. 1998: Sawfly (Hymenoptera, Symphyta) community in the Devínska Kobyla National Nature Reserve. - Biologia, Bratislava 53(2): 213-221.

Roller, L. 1999a: Spoločenstvá hrubopásych (Hymenoptera: Symphyta) vybraných zoogeografických regiónov Slovenska. PhD thesis, Ústav zoológie, Slovenská akadémia vied, Bratislava, 180 pp.

Roller, L. 1999b: First records of Nematinae (Hymenoptera, Symphyta, Tenthredinidae) in Slovakia. -Biologia, Bratislava 54(5): 599-600.

Roller, L. 1999c: Faunistic records from Slovakia. Hymenoptera: Symphyta: Tenthredinidae: Nematinae. -Entomological Problems 30(1): 30.

Roller, L. 1999d: Faunistic records from Slovakia. Hymenoptera: Symphyta: Tenthredinidae: Nematinae. -Entomological Problems 30(1): 52.

RoLler, L. 1999e: Check list of the sawflies (Hymenoptera: Symphyta) of Slovakia. -Entomological Problems 30(2): 37-48.

Roller, L. 2000a Zubačkovité (Megalodontesidae) - hrubopáse xerotermov. Hmyz 1(1): 17-18.

Roller, L. 2000b First records of Blasticotomidae, Tenthredinidae, Pamphiliidae (Hymenoptera) from Slovakia. -Biologia, Bratislava 55(5): 561-562.

Roller, L. 2000c Súčasný stav poznania fauny hrubopásych (Hymenoptera, Symphyta) na Slovensku. -Správy Slovenskej zoologickej spoločnosti 18: 109-114.

Roller, L. 2001: Príspevok k poznaniu hrubopásych (Hymenoptera, Symphyta) a rohačkovitých (Diptera, Sciomyzidae) prírodnej pamiatky Mitická slatina. p. 32-36. In: Májsky, J. (ed.), Zborník výsledkov inventarizačného výskumu prírodnej pamiatky Mitická slatina. Občianske združenie Pre Prírodu, Trenčín, 99 pp.

Roller, L. 2004. Hrubopáse blanokrídlovce (Hymenoptera, Symphyta) Tematínskych kopcov. -Entomofauna Carpathica 16: 56-64.

Roller, L. 2005: Blanokrídlovce (Hymenoptera): hrubopáse (Symphyta). 117-123 In: Fauna Devínskej Kobyly. APOP, Bratislava, $181 \mathrm{pp}$.

Roller, L. 2006a: Seasonal flight activity of sawflies (Hymenoptera, Symphyta) in submontane region of the West Carpathians, Central Slovakia. -Biologia, Bratislava 61(2): 193-205.

Roller, L. 2006b: Hrubopáse blanokrídlovce (Hymenoptera, Symphyta) Tematínskych vrchov - zhrnutie faunistického výskumu. p. 53-55. In: K. Rajcová (ed.): Najvzácnejšie prírodné hodnoty Tematínskych vrchov. Zborník výsledkov inventarizačného výskumu územia európskeho významu Tematínske vrchy. KOZA, Trenčín a Pre Prírodu, Trenčín, 101 pp.

Roller, L. \& Haris, A. 2008: Sawflies of the Carpathian Basin, History and Current Research. - Natura Somogyiensis 11. Kaposvár, 261. pp.

Roller, L. \& Lukáš, J., 1999: New records of sawflies (Hymenoptera, Symphyta) in Slovakia. -Biologia, Bratislava 54(2): 225-228.

Roller L., Beneš K., Blank S. M., Holuša J., Jansen E., Jänicke M., Kaluza S., Kehl A., Kehr I., Kraus M., Liston A. D., Nyman T., Nie H., Savina H., Taeger A. \& Wei M., 2006: Contribution to the knowledge of sawfly fauna (Hymenoptera, Symphyta) of the Low Tatras National Park in Central Slovakia. -Naturae Tutela 10: 57-72.

Zhelochovtsev, A. N. 1988: Otryad Hymenoptera - Pereponchatokrylye, Podotryad Symphyta Sidyachebryukhie, 7-234. In: Medvedev, K.H. (ed.) Opredelitel nasekomykh evropeiskoi chasti SSSR, Vol. 3 Hymenoptera, Part 6, Nauka, Leningrad.

ZomвoRI, L. 1982: Tenthredinoidea - Levéldarázs-alkatúak II. -In: Fauna Hungariae, Akadémiai Kiadó, Budapest, 153, 11(3/A), $144 \mathrm{p}$.

Zombori, L. 1990: Tenthredinoidea - Levéldarázs-alkatúak III. -In: Fauna Hungariae, Akadémiai Kiadó, Budapest, 165, 11(3/B), 81 pp. 